\title{
Evaluation of adipose-derived stem cells for tissue- engineered muscle repair construct-mediated repair of a murine model of volumetric muscle loss injury
}

This article was published in the following Dove Press journal:

International Journal of Nanomedicine

8 April 2016

Number of times this article has been viewed

\author{
Venu Kesireddy ${ }^{1,2}$ \\ 'Wake Forest Institute for \\ Regenerative Medicine, Wake Forest \\ University Baptist Medical Center, \\ Winston Salem, NC, USA; ${ }^{2}$ Center \\ for Craniofacial Research, School of \\ Dentistry, University of Texas Health \\ Science Center at Houston, Houston, \\ TX, USA
}

\begin{abstract}
Volumetric muscle loss (VML) can occur from congenital defects, muscle wasting diseases, civilian or military injuries, and as a result of surgical removal of muscle tissue (eg, cancer), all of which can lead to irrevocable functional and cosmetic defects. Current tissue engineering strategies to repair VML often employ muscle-derived progenitor cells (MDCs) as one component. However, there are some inherent limitations in their in vitro culture expansion. Thus, this study explores the potential of adipose-derived stem cells (ADSCs) as an alternative cell source to MDCs for tissue engineering of skeletal muscle. A reproducible VML injury model in murine latissimus dorsi muscle was used to evaluate tissue-engineered muscle repair (TEMR) constructs incorporating MDCs or ADSCs. Importantly, histological analysis revealed that ADSC-seeded constructs displayed regeneration potential that was comparable to those seeded with MDCs 2 months postrepair. Furthermore, morphological analysis of retrieved constructs demonstrated signs of neotissue formation, including cell fusion, fiber formation, and scaffold remodeling. Immunohistochemistry demonstrated positive staining for both structural and functional proteins. Positive staining for vascular structures indicated the potential for longterm neotissue survival and integration with existing musculature. Qualitative observation of lentivirus-Cherry-labeled donor cells by immunohistochemistry indicates that participation of ADSCs in new hybrid myofiber formation incorporating donor cells was relatively low, compared to donor MDCs. However, ADSCs appear to participate in vascularization. In summary, I have demonstrated that TEMR constructs generated with ADSCs displayed skeletal muscle regeneration potential comparable to TEMR-MDC constructs as previously reported.

Keywords: skeletal muscle regeneration, muscle-derived progenitor cells, immunomodulation, paracrine signaling
\end{abstract}

\section{Introduction}

Although regeneration occurs throughout the animal kingdom, there are large disparities in the degree of inherent regeneration capacity not only among species but also amid tissue types. ${ }^{1,2}$ The field of regenerative medicine seeks to supplement or enable the regenerative process across a variety of human tissues, thereby compensating for limitations inherent in the self-repair potential of many critical organs and systems. ${ }^{3}$ Though skeletal muscle possesses a rather remarkable capacity for self-regeneration in response to smaller injuries, ${ }^{4,5}$ disease, congenital defects, surgical side effects, and trauma may all result in permanent defects in the appearance and, more importantly, function of skeletal muscle. ${ }^{6,7}$ Deficits in this category, known as volumetric muscle loss (VML), ${ }^{8}$ cannot be restored with existing therapies, including surgical repair with flaps ${ }^{9}$ and physical therapy. ${ }^{8}$ Therefore, regenerative medicine technologies to
Correspondence: Venu Kesireddy Center for Craniofacial Research, Behavioral and Biomedical Sciences Building, School of Dentistry, University of Texas Health Science Center at Houston, 194I East Road, Houston, TX 77054, USA

Tel + I 7134862573

Fax + I 7I3 4864372

Email Venu.Kesireddy@uth.tmc.edu 
treat these injuries would be of great value, as the current standard of care for VML injuries is extremely poor.

Approaches in this vein currently under development include those employing an acellular scaffold, ${ }^{10,11}$ stem or progenitor cells, ${ }^{12-15}$ or a combination of both. ${ }^{16-18}$ Several groups have reported variable neotissue formation and functional recovery in skeletal muscle injuries by use of either satellite cell-derived muscle progenitor cells ${ }^{19}$ or mesenchymal stem cells (MSCs) ${ }^{20-23}$ In contrast to these approaches, recent reports ${ }^{16,17}$ have focused on VML injury repair with tissueengineered muscle repair (TEMR) constructs generated by seeding muscle-derived progenitor cells (MDCs) on bladder acellular matrix (BAM) scaffolds and subjecting them to in vitro differentiation and maturation in a bioreactor. Although these studies elegantly demonstrated the utility of TEMR for the treatment of VML injuries in flat sheet-based muscles such as latissimus dorsi (LD) in rodents, ${ }^{16}$ several obstacles to scaling up the technology still remain for successful application of TEMR constructs to large injuries in humans, especially complex traumatic injuries sustained both on and off the battlefield. VML injuries often result in loss of not only muscle tissue but also the supporting infrastructure such as accompanying blood vessels and nerve connections. ${ }^{8}$ It is conceivable that to reconstruct such large injuries, far greater number of muscle stem and progenitor cells are required. However, due to the limited size of biopsies that can be used for muscle progenitor cell isolation, there is a significant need for in vitro culture expansion. The problem is further compounded by the difficulty inherent in culture expansion of muscle progenitor cells while still maintaining their myogenic phenotype. ${ }^{24,25}$

Stem cells offer an attractive alternative. To date, a host of stem cell types have been evaluated in muscle repair applications, including embryonic stem cells, ${ }^{13,26}$ pluripotent adult stem cells, ${ }^{22,27}$ muscle resident side population cells, ${ }^{14}$ bone marrow-derived stem cells, ${ }^{20,22}$ stromal cells isolated from synovial membrane, ${ }^{21}$ human umbilical cord-derived cells, ${ }^{28}$ pericytes,${ }^{15}$ and meso-angioblasts. ${ }^{29}$

In this study, we evaluated the potential of adiposederived stem cells (ADSCs) as an alternative cell source for tissue-engineered skeletal muscle to circumvent the limited scaling ability of MDCs. ADSCs represent an abundant, easy to expand, pluripotent adult stem cell source that can be easily isolated from adipose tissue stromal vascular fraction and compares favorably to bone marrow-derived MSCs in terms of ease of access. ${ }^{30,31}$ Similar to bone marrow-derived MSCs, adipose tissue stromal vascular fraction-derived ADSCs have also been shown to differentiate into chondrogenic, osteogenic, adipogenic, and myogenic lineages. ${ }^{30,31}$ Extant literature also documents their capacity for differentiation toward nonmesenchymal lineages such as neurons $\mathrm{s}^{32}$ and hepatocytes. Moreover, literature evidence suggests that soluble factors secreted by myogenic cells are sufficient to promote the expression of muscle-specific proteins in ADSCs cocultured with myogenic cells, ${ }^{33}$ and uncultured ADSCs were shown to possess an intrinsic myogenic potentia ${ }^{33}$ albeit at a very low efficiency. Some of the aforementioned cell types have demonstrated great promise when injected into injured muscles; however, none of these studies investigated the potential of ADSCs in the repair of clinically relevant larger defects modeling VML injuries. Therefore, this study forms a logical extension of the findings by Machingal et al ${ }^{16}$ with TEMR constructs by exploring the potential of TEMR-ADSC constructs for repair of VML injuries created in a murine LD muscle.

\section{Materials and methods Experimental animals}

All animals were purchased from commercial vendors (Harlan Laboratories, Indianapolis, IN, USA). Male Lewis rats of age 3-4 weeks or 8-10 weeks were used for isolation of donor MDCs or ADSCs, respectively. Female athymic nude mice $(\mathrm{nu} / \mathrm{nu})$ of age $8-10$ weeks were used for in vivo studies of VML injury repair. All animal procedures were approved by the Institutional Animal Care and Use Committee at Wake Forest University and were performed in accordance with animal care and use guidelines set by the American Physiological Society.

\section{Isolation of MDCs and ADSCs}

MDCs were isolated from soleus and tibialis anterior muscles of donor rats. Isolated muscle tissue was cleared of adventitious tissues and tendons and minced into small pieces. Minced muscle tissue was incubated in $0.2 \%$ collagenase type I (Worthington Biochemicals, Lakewood, NJ, USA) in Dulbecco's Modified Eagle's Medium (DMEM) low glucose (Hyclone, Logan, UT, USA) for 2 hours at $37^{\circ} \mathrm{C}$ followed by neutralization with complete medium containing DMEM low glucose supplemented with 20\% fetal bovine serum (FBS) (Hyclone), 10\% horse serum (Hyclone), 1\% chicken embryo extract (Accurate Chemical \& Scientific Corporation, Westbury, NY, USA), and 1\% penicillin/streptomycin (Thermo Fisher Scientific, Waltham, MA, USA). Digested muscle fibers were plated onto tissue culture dishes coated with MatrigelTM (BD Biosciences, San Jose, CA, USA) in complete myogenic medium. Matrigel was diluted 1:50 in DMEM for coating tissue culture plates. Cells were passaged at $60 \%$ confluence, cultured in seeding medium containing DMEM low glucose supplemented with $15 \% \mathrm{FBS}$ and $1 \%$ penicillin/streptomycin, and used for seeding at passages not $>2$. 
ADSCs were isolated following the protocols described in the literature. ${ }^{31,33}$ Briefly, adipose tissue collected from visceral or subcutaneous regions from donor rats was washed extensively with DMEM low glucose supplemented with $10 \%$ antibiotic/antimycotic solution (Thermo Fisher Scientific). Washed tissue was minced and digested with $0.2 \%$ collagenase type I in DMEM at $37^{\circ} \mathrm{C}$ for 1 hour with constant shaking. Following complete digestion, adipose tissue slurry was neutralized with complete medium consisting of DMEM low glucose supplemented with $10 \% \mathrm{FBS}$ and $1 \%$ antibiotic/antimycotic and centrifuged to separate the fatty top layer (consisting of mature adipocytes) from a pellet consisting of a heterogeneous population of cells including ADSCs. Pelleted cells were resuspended and filtered through a $100 \mu \mathrm{m}$ cell strainer (BD Biosciences) and plated on tissue culture dishes.

\section{Preparation of BAM scaffolds}

BAM scaffolds were prepared from porcine urinary bladder as previously described. ${ }^{34}$ Briefly, bladders were washed extensively, trimmed, and microdissected to obtain the lamina propria layer and decellularized in $0.05 \%$ trypsin (Hyclone) for 1 hour at $37^{\circ} \mathrm{C}$. Decellularized bladders were then neutralized overnight with DMEM low glucose supplemented with $10 \% \mathrm{FBS}$ and $1 \%$ penicillin/streptomycin. Neutralized bladders were further processed by washing with 1\% Triton X-100 (Sigma-Aldrich Co., St Louis, MO, USA) and treating with $0.1 \%$ ammonium hydroxide (SigmaAldrich Co.) in deionized water for 3 days at $4^{\circ} \mathrm{C}$ followed by a final wash with deionized water for 3 days at $4^{\circ} \mathrm{C}$. Structural composition and absence of cellular elements were confirmed by histological assessments. The decellularized lamina propria was further peeled to obtain a thin urothelial layer of BAM scaffold of 0.3-0.4 mm thickness. The prepared BAM scaffold was cut into strips of $3 \mathrm{~cm} \times 2 \mathrm{~cm}$ dimensions and draped over precut medical grade silicone molds (McMaster-Carr, Atlanta, GA, USA). Finally, scaffolds with silicone molds were freeze-dried in a benchtop freeze-dryer (Labconco, Kansas City, MO, USA) and sterilized by ethylene oxide gas sterilizer (Anderson Products, Haw River, NC, USA) and stored at room temperature until further use. Immediately prior to cell seeding, scaffolds were prewetted with sterile phosphate-buffered saline (PBS) for 30 minutes at $37^{\circ} \mathrm{C}$, and then ADSCs or MDCs were seeded at 1 million cells $/ \mathrm{cm}^{2}$ of scaffold area.

\section{Characterization of donor cells by immunofluorescence analysis}

Isolated ADSCs and MDCs (at passage 2) were characterized for the presence of standard cell surface markers or nuclear markers, respectively, by seeding them on uncoated chamber slides or coverslips at 4,000 cells $/ \mathrm{cm}^{2}$ of area and cultured for 3 days. MDC-seeded coverslips were fixed with $4 \%$ paraformaldehyde (PFA) in PBS and permeabilized with $0.5 \%$ Triton-X-100, whereas ADSC-seeded coverslips were fixed with 4\% PFA in PBS but not permeabilized for evaluation of surface markers (CD73, CD90, CD34, CD45, CD31, Sca I) but permeabilized when testing for the absence of muscle-specific nuclear markers (Pax7, MyoD, and myogenin). Fixed and/or permeabilized coverslips were blocked with 3\% bovine serum albumin in PBS for 30 minutes and later incubated with primary antibodies diluted in PBS for 1 hour (Table 1). Anti-mouse secondary antibodies made in either goat or horse and conjugated to a Texas Red fluorophore were diluted 1:200 in PBS and incubated with primary labeled coverslips for 30 minutes at room temperature. Fluorescence microscopy images were obtained with a Zeiss Axio imager M1 (Carl Zeiss Microscopy, Thornwood, NY, USA) or DM4000B Leica upright microscope (Leica Microsystems, Wetzlar, Germany).

Table I Surface and nuclear marker expression in cultured ADSCs and MDCs

\begin{tabular}{|c|c|c|c|c|c|c|}
\hline Marker & Source & Class & Made in & $I^{0}$ dilution & ADSCs & MDCs \\
\hline \multicolumn{7}{|l|}{ Surface markers } \\
\hline CD73 & BD Biosciences & $\lg G \mid$ & Mouse & I:10 (FACS); I:200 (IF) & $+\mathrm{ve}$ & NT \\
\hline CD90 & BD Biosciences & $\lg G \mid$ & Mouse & I:10 (FACS); I:200 (IF) & +ve & NT \\
\hline CD34 & Novus Biologicals & $\lg G \mid$ & Mouse & I:10 (FACS); I:200 (IF) & $-\mathrm{ve}$ & NT \\
\hline Sca I & Abcam & $\lg G 2 a$ & Mouse & I: I0 (FACS); I:200 (IF) & $-v e$ & NT \\
\hline \multicolumn{7}{|l|}{ Nuclear markers } \\
\hline $\operatorname{Pax} 7$ & DHSB & $\lg G \mid$ & Mouse & I:I0 (FACS); I:50 (IF) & $-v e$ & + ve \\
\hline MyoD & Dako Denmark A/S & $\lg G \mid$ & Mouse & I:I0 (FACS); I:50 (IF) & $-\mathrm{ve}$ & +ve \\
\hline Myogenin (F5D) & DHSB & $\lg G \mid$ & Mouse & I:I0 (FACS); I:50 (IF) & $-v e$ & $+\mathrm{ve}$ \\
\hline
\end{tabular}

Notes: Source details: BD Biosciences (San Jose, CA, USA); Novus Biologicals (Littleton, CO, USA); Abcam (Cambridge, UK); DHSB (lowa City, IA, USA); Dako Denmark A/S (Glostrup, Denmark).

Abbreviations: CD, cluster of differentiation; Sca I, stem cell antigen I; lgG, immunoglobulin G; $1^{1}$, primary antibody; ADSCs, adipose-derived stem cells; MDCs, musclederived progenitor cells; FACS, fluorescence-activated cell sorting; IF, immunofluorescence; NT, not tested; +ve, positive; -ve, negative. 


\section{Characterization of donor cells via fluorescence-activated cell sorting}

ADSCs or MDCs (passage 2) were prepared for fluorescenceactivated cell sorting (FACS) analysis by resuspending them at 1 million cells $/ 100 \mu \mathrm{L}$ in blocking buffer $(10 \%$ FBS in PBS). For surface marker analysis, ADSCs were incubated with primary antibodies $(1: 10$, dilution in $100 \mu \mathrm{L}$ of blocking buffer) for 30 minutes (Table 1) and then washed twice with $10 \%$ sodium azide in blocking buffer. Cells were then incubated with anti-mouse secondary antibodies made in either goat or horse and conjugated to a fluorescein isothiocyanate fluorophore for 30 minutes, washed twice in blocking buffer, and postfixed using 2\% PFA in blocking buffer for 15 minutes. For nuclear marker analysis, ADSCs and MDCs were prepared by fixing with 2\% PFA in blocking buffer and then permeabilized with permeabilization buffer consisting of $0.2 \%$ bovine serum albumin $+0.15 \%$ Triton-X-100 in Tris-buffered saline. Cells were then incubated with primary antibodies (Table 1) for 1 hour and followed by washing twice with block buffer and incubation with anti-mouse secondary antibodies made in either goat or horse and conjugated to fluorescein isothiocyanate fluorophore for 30 minutes. Prepared cells were acquired by BD FACSCalibur flow cytometer (BD Biosciences) using $488 \mathrm{~nm}$ laser and analyzed with CellQuest Pro software version 3.3 (BD Biosciences).

\section{Experimental design}

\section{Preparation of TEMR constructs}

TEMR constructs were created by seeding either MDCs (TEMR-MDCs) or ADSCs (TEMR-ADSCs) on BAM scaffolds. While MDC-seeded BAM scaffolds underwent in vitro differentiation and maturation in bioreactors as previously described ${ }^{16}$ before implantation, ADSC-seeded BAM scaffolds were kept in ADSC proliferation media for 3 days but did not undergo any differentiation protocols before implantation. Both sides of the scaffold were seeded with cells by flipping the scaffolds a day after the initial seeding. The experimental groups tested included R-TEMR-ADSCs $(\mathrm{n}=13), \mathrm{R}-\mathrm{TEMR}-\mathrm{MDCs}(\mathrm{n}=10)$, scaffold only (R-S, $\mathrm{n}=8)$, injury but no repair ( $N R, n=7)$, and uninjured native LD muscles.

\section{Surgical creation of VML injury and TEMR construct implantation}

Surgical creation of the murine VML injury and implantation of TEMR constructs from experimental groups were performed as previously reported. ${ }^{16} \mathrm{VML}$ injury was surgically created as a critical size defect $(\approx 50 \%)$ of the LD muscle in isoflurane-anesthetized $\mathrm{nu} / \mathrm{nu}$ mice using a previously reported methodology. ${ }^{16}$ Briefly, a longitudinal incision was made along the midline of the back. The trapezius muscle that covers the LD muscle was lifted to expose the LD muscle without removing the tendon inserted at the humerus. Suture markers were then placed on the LD muscle demarking the superior half of the spinal fascia and the medial half of the muscle head at the humerus. The medial half of the muscle was then excised using a fine scissor. Using this methodology, a defect weighing $\approx 16-20 \mathrm{mg}$ was excised from the LD muscle. After surgical excision, mice were implanted with either R-TEMR-ADSCs or R-TEMR-MDCs constructs. Scaffold only (R-S), injury but no repair (NR), and uninjured native LD muscles formed other groups. The TEMR constructs were sutured to the remaining LD muscle at the site of injury using Vicryl 6-0 sutures. In all cases, the fascia and skin were then sutured closed, and the animals were allowed to recover from anesthesia. Animals were sacrificed, and tissue-engineered LD muscles (consisting of remaining native muscle and TEMR constructs) were retrieved 2 months postimplantation for histological evaluation.

\section{Histology and immunohistochemistry}

Retrieved LD muscles were processed for paraffin embedding using standard methods. Briefly, LD muscles were fixed in $10 \%$ neutral buffered formalin ( $10 \times$ tissue volume) overnight, changed into $60 \%$ ethanol solution, and subsequently processed in ASP300S paraffin tissue processor (Leica Microsystems) and embedded into paraffin blocks in tissue embedding cassettes (Electron Microscopy Sciences, Hatfield, PA, USA). Masson's trichrome staining and immunohistochemical (IHC) stainings were performed on 7-10 $\mu \mathrm{m}$ thick serial sections cut from the paraffin-embedded blocks on a Leica RM 2135 microtome (Leica Microsystems). IHC stainings were performed using antibodies to detect myosin (MF-20, 1:10), titin (9D10, 1:10), ryanodine receptor 1 (RyR1; 34C, 1:10), and Junctophilin 1 (Jp1; Thermo Fisher Scientific 40-5100, 1:120). MF-20, titin, and RyR1 antibodies were acquired from Developmental Studies Hybridoma Bank, created by the National Institute of Child Health and Human Development of the National Institutes of Health and maintained at The University of Iowa, Department of Biology, Iowa City, IA, USA. All the remaining reagents and secondary antibodies used for IHC staining were obtained from Vector Laboratories Inc., Burlingame, CA, USA. Biotinylated anti-mouse IgG secondary antibody (MKB$2225,1: 250)$ or anti-rabbit secondary antibody (BA-1000, 1:500) was used to detect mouse (MF-20, 9D10, and RyR1) 
or rabbit (Jp1) primary antibodies. The color development was done by treating the antibody-labeled sections with avidin biotin complex reagent (PK-7100) and then visualized using ImmPACT ${ }^{\mathrm{TM}}$ NovaRED ${ }^{\mathrm{TM}}$ substrate kit (SK-4800). Finally, the sections were counterstained using Gill's hematoxylin (GHS280; Sigma-Aldrich Co.). Images were captured and digitized using DM4000B Leica Upright Microscope (Leica Microsystems) equipped with QICAM digital camera (QImaging, Surrey, BC, Canada) at different magnifications.

\section{Detection of donor cells by immunohistochemistry}

Lentivirus expressing Cherry reporter was transduced into either MDCs or ADSCs prior to seeding onto BAM scaffolds. TEMR-lenti-Cherry-MDC/ADSC constructs were implanted at the site of surgical repair, and engineered muscles were retrieved 2 months postrepair and processed for paraffin embedding. IHC staining was performed on sections with antiCherry antibody (Novus Biologicals, Littleton, CO, USA).

\section{Results}

\section{Characterization of ADSCs and MDCs}

ADSCs isolated from donor rats (passage 2) were characterized for the presence of standard positive and negative markers by immunofluorescence microscopy and FACS analysis (Figure 1). It was evident from FACS analysis that ADSCs expressed standard surface markers CD73 and CD90 but did not express CD34, CD31, CD45 (data not shown), and Sca I, confirming the relative purity of isolated ADSCs. ADSCs also did not express standard nuclear muscle markers Pax 7 (data not shown), MyoD, and myogenin, ruling out any precontamination of ADSCs with muscle progenitor cells. FACS analysis estimated the percentage of CD73 and CD90 cells as $89.42 \% \pm 2.4 \%$ and $92.80 \% \pm 2 \%$, respectively. Figure $1 \mathrm{H}$ indicates the average of CD73 and CD90 percentages from three separate experiments. Characterization of MDCs displayed a heterogeneous population of cells representing satellite cells (stem cells, Pax7 +ve [16.36\% $44 \%]$ ), progenitor cells (MyoD +ve, 31.32\% $14 \%$ ), and differentiationcommitted cells (myogenin +ve, 20.91\% $2 \%$ ), thus, essentially comprising cells at various developmental stages of cell cycle (Table 1).

\section{Histological characterization of TEMR construct-repaired muscles and evidence for neotissue formation}

Gross morphology and qualitative assessment of neotissue formation and extent of scaffold remodeling were assessed in VML injuries repaired with TEMR-MDC (Figure 2) or TEMR-ADSC (Figure 3) constructs, 2 months postrepair, by Masson's trichrome staining. Figure 2A depicts the interface of retrieved engineered muscle repaired with TEMR-MDC constructs. The VML injury repair with TEMR-MDC constructs exhibited typical hallmarks for neotissue formation such as fusion of myoblasts (Figure 2D), differentiated elongated fiber formation (Figure 2C), and smaller mature striated myofibers (Figure 2B) on the scaffold part of the engineered muscle reminiscent of in vitro differentiation of skeletal muscle cells. These observations provide compelling evidence for new tissue formation in TEMR-MDC constructs. VML injury repairs with TEMR-ADSC constructs similarly displayed robust regeneration potential as indicated by the remodeling of the scaffold (arrows in Figure 3A and $\mathrm{C}$ ) and apparent regenerating new myofibers (arrows in Figure 3B and D). The nascent myofibers are relatively thin and short compared to native myofibers (Figure 3B). The presence of blood vessels is marked by \# in Figure 3D, indicating the presence of vascular elements.

\section{Functional protein expression}

Immunohistological stainings were performed on tissue sections of paraffin-embedded retrieved tissue-engineered muscles repaired with TEMR-ADSC constructs. Figure 4 depicts IHC stainings at the interface of native muscle and TEMR construct. Positive staining for myosin heavy chain (MF20 antibody) in a sarcomeric pattern was detected as indicated in Figure 4A. Similarly, positive staining for sarcomeric protein titin (9D10 antibody) was detected in Figure 4B. Likewise, the presence of Junctophilin 1 (Jp1) and ryanodine receptors (RyR1), two proteins that participate in excitation-contraction coupling, was also detected in Figure $4 \mathrm{C}$ and D at the interface and inside the scaffold similar to native muscles.

\section{Evidence for the presence of vascular elements in TEMR-ADSC-repaired constructs}

LD muscles repaired with TEMR-ADSC constructs were retrieved 2 months postimplantation and analyzed for the presence of vascular structures. Figure $5 \mathrm{~A}$ and B depict the interface of native LD muscle and scaffold side seeded with ADSCs at lower magnification and higher magnification, respectively. Positive staining for von Willebrand factor that stains blood vessels confirms the development of vascular elements on the scaffold side of the engineered tissue indicating the potential of long-term neotissue survival and integration with host tissue. 

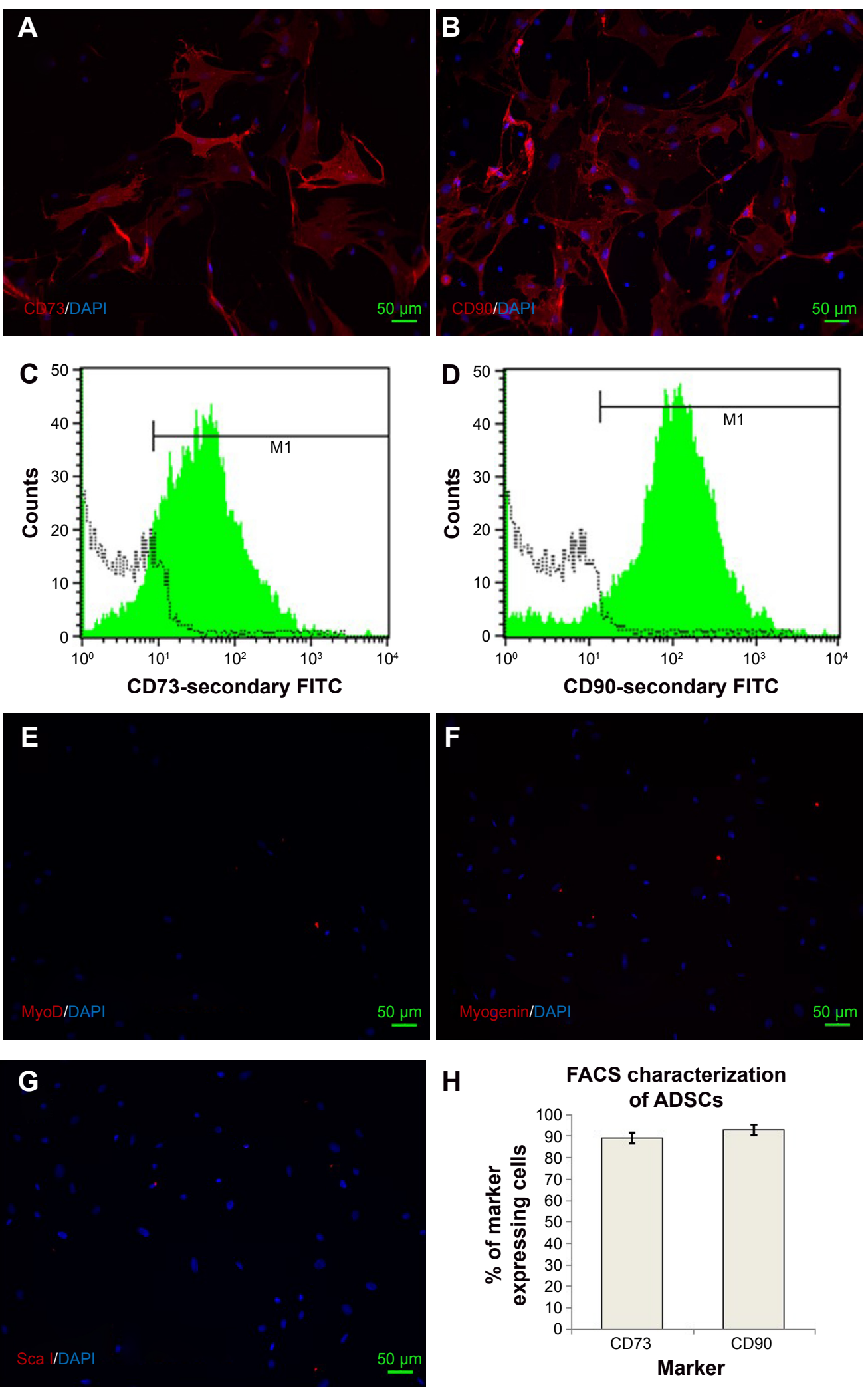

H

FACS characterization of ADSCs

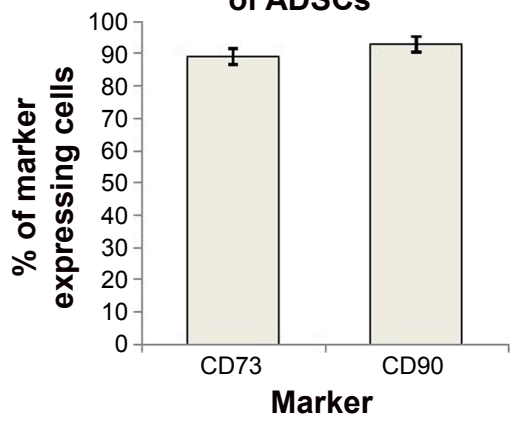

Figure I Characterization of rat adipose-derived stem cells (ADSCs) in culture.

Notes: ADSCs were cultured after isolation and cells at passage 2 were either seeded on glass coverslips and cultured in proliferation media in an undifferentiated state for 3 days or processed for FACS analysis. Expression of standard surface markers for ADSCs was confirmed by staining of CD73 (A) and CD90 (B). C and D depict percentage of CD73 and CD90 cells by FACS analysis. Absence of any contaminating muscle progenitor cells in ADSC cultures was revealed by the absence of IF staining for nuclear muscle markers MyoD $(\mathbf{E})$, myogenin $(\mathbf{F})$, and a nonspecific ADSC surface marker Sca I (G). FACS analysis $(n=3)$ estimated that $89.42 \% \pm 3.2 \%$ of cells expressed CD73, whereas CD90 was expressed by $92.80 \% \pm 2.4 \%$ cells $(\mathbf{H})$. FACS analysis also confirmed the negative staining of muscle markers (Pax7, MyoD, and myogenin) and absence of nonspecific surface marker (Sca I) in ADSCs. MI, represents gate used to define the positive cell population. Data is presented as mean \pm SD.

Abbreviations: FACS, fluorescence-activated cell sorting; FITC, fluorescein isothiocyanate; DAPI, 4', 6-diamidino-2-phenylindole. 

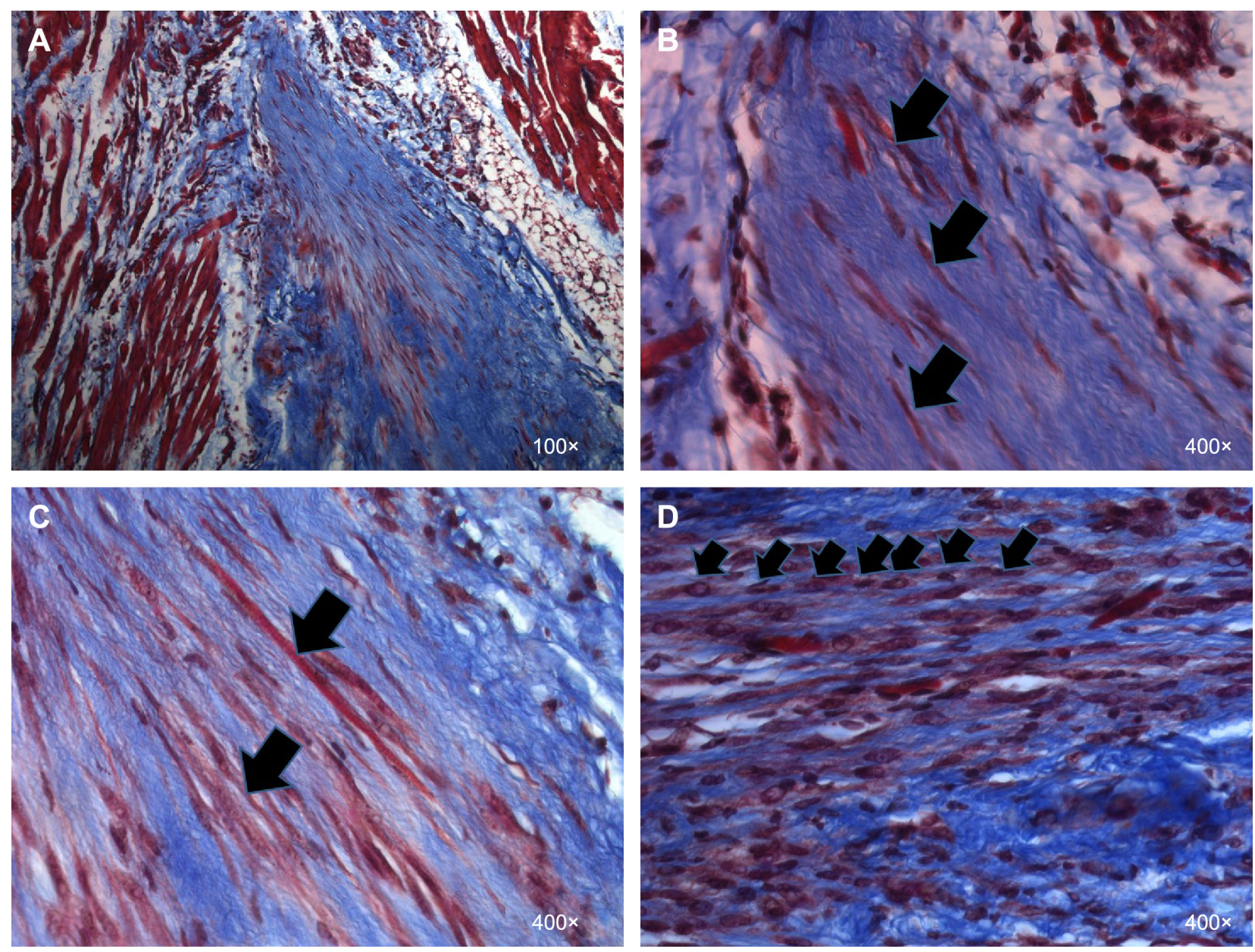

Figure 2 Evidence for new tissue formation in VML injuries repaired with TEMR-MDC constructs.

Notes: LD muscles repaired with TEMR-MDC $(n=10)$ constructs were retrieved 2 months postimplantation, paraffin embedded and processed for Masson's trichrome staining, and analyzed for morphology and new tissue formation. Red indicates muscle, blue indicates collagen, and black indicates nuclei in Masson's trichrome staining. (A) An overview at the interface of native tissue and scaffold. (B) Arrows indicate regenerating striated muscle fibers. (C) Arrows indicate long, differentiated MDCs and neofibers on the scaffold, whereas arrows in (D) indicate fusion of MDCs to form new muscle fibers.

Abbreviations: VML, volumetric muscle loss; TEMR, tissue-engineered muscle repair; MDC, muscle-derived progenitor cell; LD, latissimus dorsi.

\section{Donor cell survival and contribution to new muscle tissue formation}

Donor cell survival after implantation of TEMR-MDC and TEMR-ADSC constructs was analyzed 2 months postrepair from retrieved engineered muscles (Figures 6 and 7). Lentivirus expressing Cherry reporter was transduced into either MDCs or ADSCs prior to seeding on to BAM scaffolds. TEMR-lenti-Cherry-MDC/ADSC constructs were implanted at the site of surgical repair, and engineered muscles were retrieved 2 months postrepair and processed for paraffin embedding. IHC staining was performed on sections with anti-Cherry antibody (Figures 6 and 7). Negative controls from adjacent sections were displayed to confirm the specificity of staining with anti-Cherry antibody (Figures 6D and 7B and D). Newly regenerating, differentiating, and elongated myofibers incorporating donor MDCs could be detected at the interface in Figure 6A, B, and C (indicated by arrows in Figure 6C). Scaffold also displayed unfused donor MDCs (indicated by white arrows in Figure 6B). Similarly, TEMR-ADSC-repaired muscle constructs also displayed hybrid myofibers incorporating donor ADSCs (Figure 7A), but their occurrence was relatively low compared to TEMRMDC constructs. However, a large part of the scaffold displayed unfused donor ADSCs (Figure 7C). Interestingly, unfused donor ADSCs were also detected surrounding putative new blood vessel-like (structures indicated by black arrows in Figure 7C) indicating that ADSCs can potentially participate or enhance vascularization.

\section{Discussion}

Injury to skeletal muscle elicits a well-characterized regenerative response beginning with an inflammatory phase dominated by invasion of macrophages, followed by activation, proliferation, differentiation, and fusion of satellite cells, 

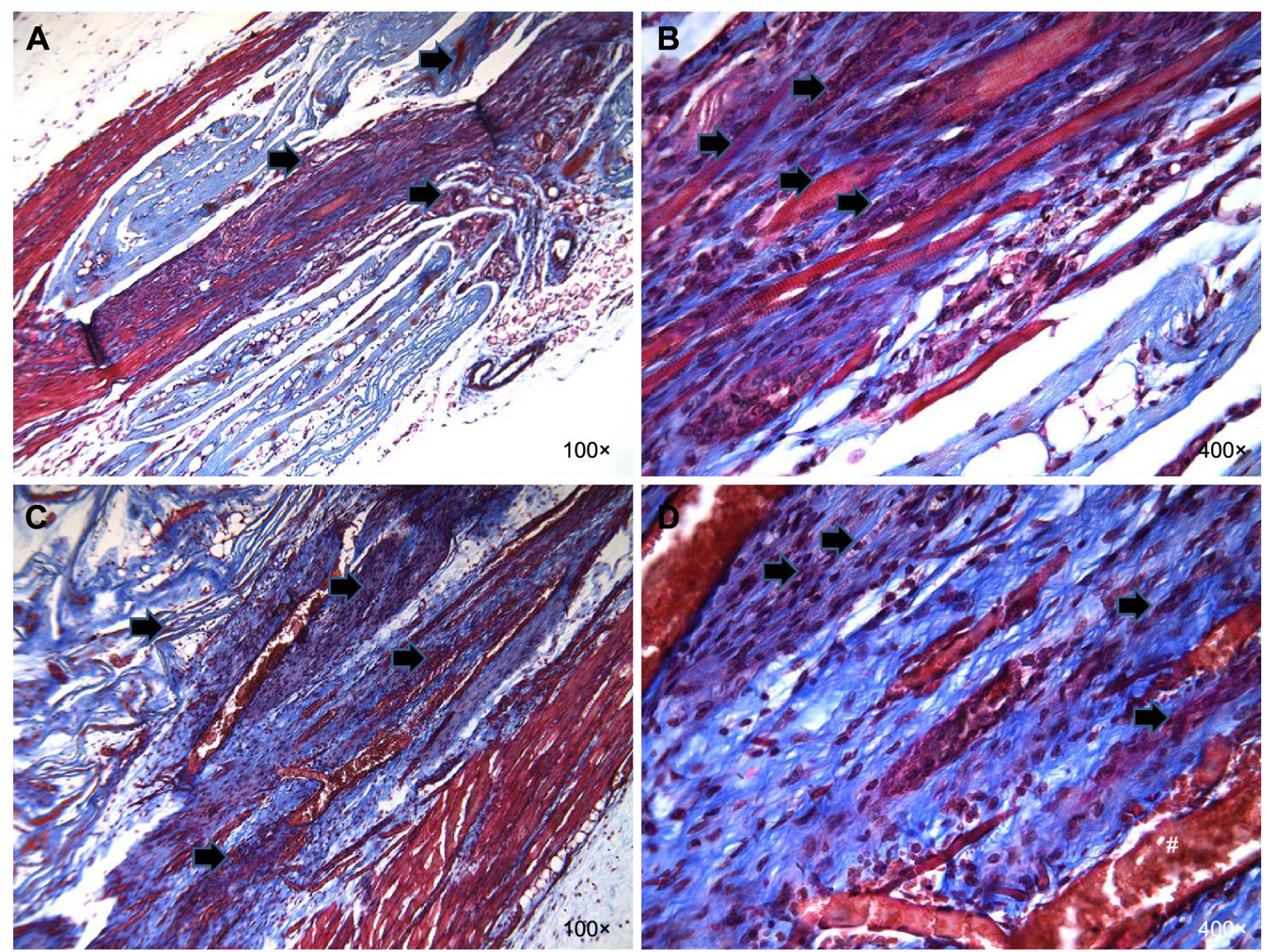

Figure 3 Evidence for new tissue formation in VML injuries repaired with TEMR-ADSC constructs.

Notes: LD muscles repaired with TEMR-ADSC $(n=13)$ constructs were retrieved 2 months postimplantation and analyzed for morphology and new tissue formation by Masson's trichrome staining on paraffin-embedded histological sections. Red indicates muscle, blue indicates collagen, and black indicates nuclei in Masson's trichrome staining. Neo tissue formation after repair with TEMR-ADSC constructs is shown in two representative animals. $\mathbf{A}$ and $\mathbf{C}$ display an overview of the native tissue and scaffold and $\mathbf{B}$ and $\mathbf{D}$ depict magnified view on the engineered side of the scaffold in two representative animals. Neo tissue formation is evident from signs of fusion, remodeling of the scaffold, and fiber formation on the scaffold part of the engineered muscle. Arrows indicate remodeling of the scaffold ( $\mathbf{A}$ and $\mathbf{C})$ or regenerating muscle fibers (B and $\mathbf{D}$ ); \# indicates the presence of vascular structures (blood vessels).

Abbreviations: VML, volumetric muscle loss; TEMR, tissue-engineered muscle repair; ADSC, adipose-derived stem cell; LD, latissimus dorsi.

and concluding with maturation of newly formed myofibers and remodeling of regenerated muscle. ${ }^{5,35}$ In many aspects, skeletal muscle regeneration recapitulates the events in embryonic myogenesis. ${ }^{5}$ However, in VML injuries, the mass of muscle lost can exceed the self-repair capability of skeletal muscle. ${ }^{8}$ In these cases, the normal regeneration process is insufficient due to its inability to reconstruct both cellular and extracellular matrix components. Instead of a mature, functioning tissue, the body responds by depositing fibrous scar tissue, ${ }^{8,36}$ resulting in both functional and cosmetic deficits. Current "gold standard" treatments involving VML repair with muscular flaps or grafts are usually associated with poor engraftment to the host tissue and significant donor site morbidity. ${ }^{9}$ This lack in standard of care, when combined with a significant patient population, represents a clear unmet clinical need.

Thus, developing tissue-engineered skeletal muscle for VML repair has become a recent priority, as this approach bypasses many limitations of autologous grafting or flap transfer. ${ }^{9}$ These approaches broadly focus on implanting a biomimetic scaffold, either with or without cells. In this category, recent reports based on TEMR constructs for repair of VML injuries in murine LD model restored functional values to $\sim 60 \%-70 \%$ of native uninjured muscle, 2 months postrepair. ${ }^{16,17}$ While both studies utilized MDCs for the generation of TEMR constructs, this study differs from the previous studies in that it seeks to explore the potential of alternative cell source, namely, ADSCs for VML injury repair with TEMR-based approach.

The rationale for this study stems from the fact that MDC expansion in culture is invariably associated with loss of myogenic capacity. Regenerating large tissue mass requires a greater number of cells, which would necessitate larger biopsy size given expansion limitations in vitro.

The primary hypothesis and novelty of this study are that MDCs can be substituted by adult stem cells such as ADSCs 

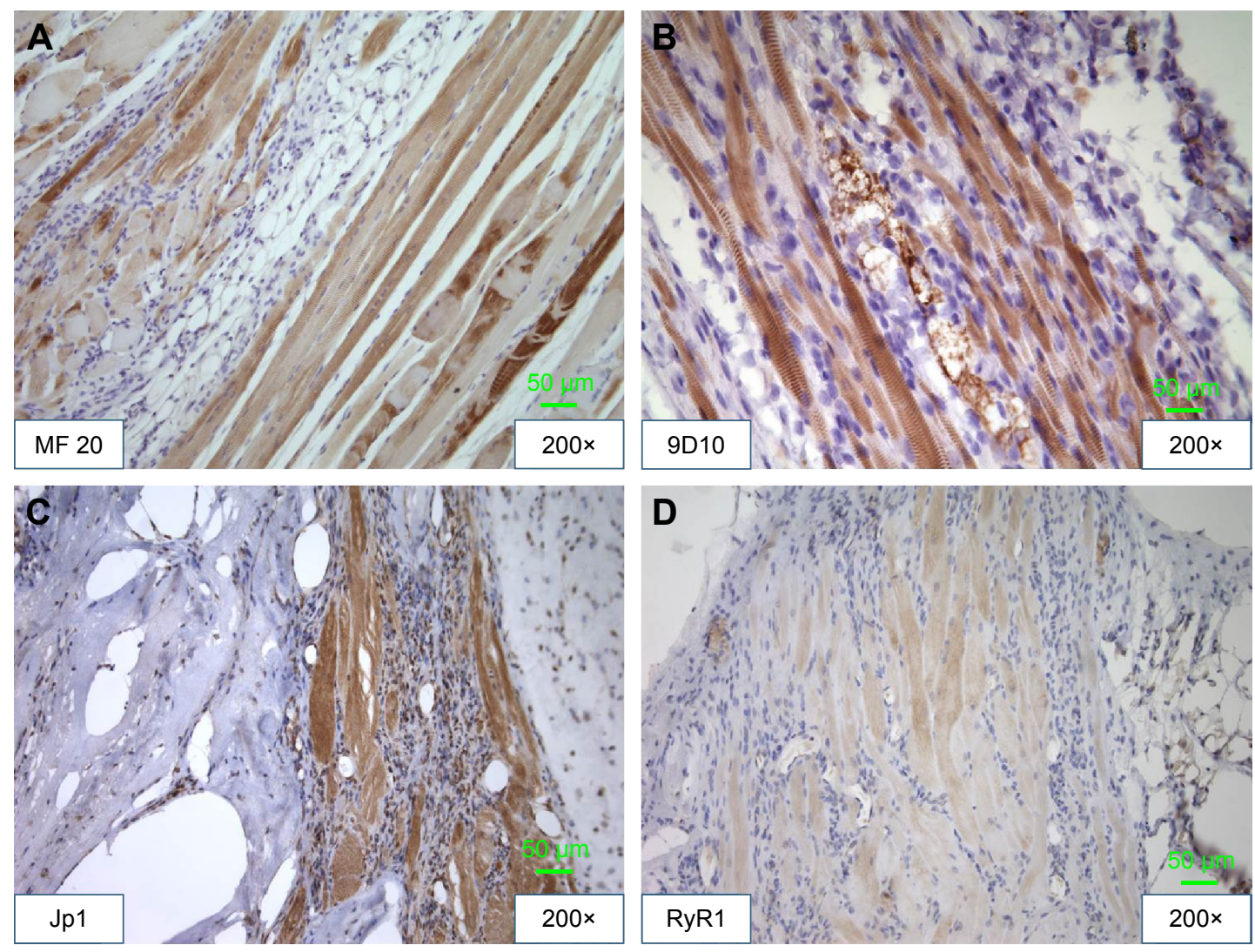

Figure 4 TEMR-ADSC construct-repaired LD muscles display structural and functional hallmarks reminiscent of native muscles.

Notes: LD muscles repaired with TEMR-ADSC constructs were retrieved 2 months postimplantation and analyzed for morphology and new tissue formation by IHC staining on paraffin-embedded histological sections. IHC staining demonstrated positivity for structural proteins, myosin heavy chain (MF20; A) and titin (9DI0; B). Similarly IHC staining revealed the presence of functional proteins, Junctophilin (JpI; C) and ryanodine receptor I (RyRI; D).

Abbreviations: TEMR, tissue-engineered muscle repair; ADSC, adipose-derived stem cell; LD, latissimus dorsi; IHC, immunohistochemistry.

in TEMR construct-mediated VML injury repairs while achieving similar regeneration potential. To this end, we compared TEMR constructs seeded with MDCs or ADSCs in their efficiency for VML repair. Prior to implantation, ADSCs were analyzed for standard cell surface markers and confirmed to be free of any contaminating muscle cells. Characterization of MDCs prior to seeding on BAM scaffolds revealed a heterogeneous population of cells representing satellite cells, progenitor cells, and differentiationcommitted cells, thus, essentially comprising cells at various
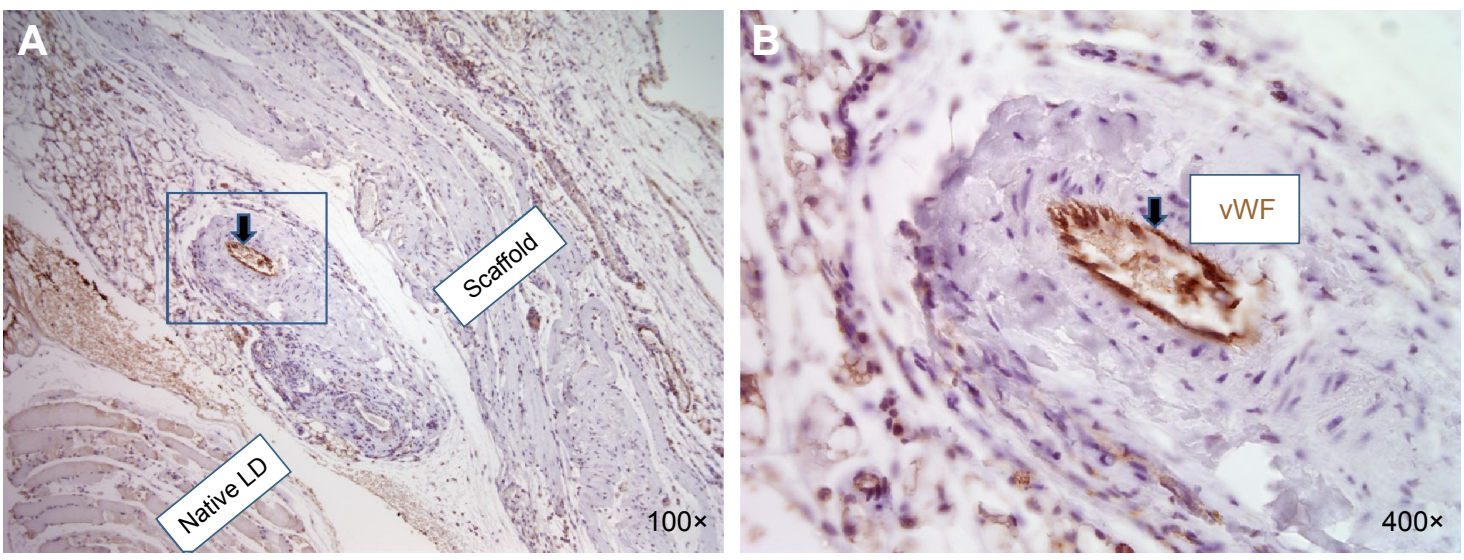

Figure 5 Evidence for the presence of vascular elements on the scaffold side in retrieved engineered muscles repaired with TEMR-ADSC constructs.

Notes: LD muscles repaired with TEMR-ADSC constructs were retrieved 2 months postimplantation and analyzed for the presence of vascular structures. The interface of native LD muscle and scaffold side seeded with ADSCs at lower magnification (A) and higher magnification (B). Positive staining for von Willebrand factor (vWF) of blood vessels confirms the development of vascular elements on the scaffold side of the engineered tissue.

Abbreviations: TEMR, tissue-engineered muscle repair; ADSC, adipose-derived stem cell; LD, latissimus dorsi. 


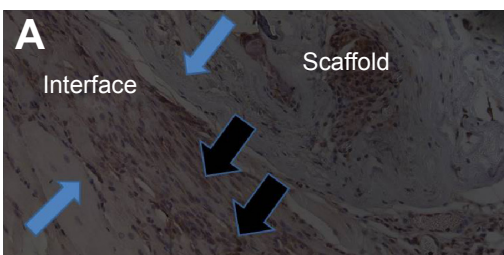

Native tissue
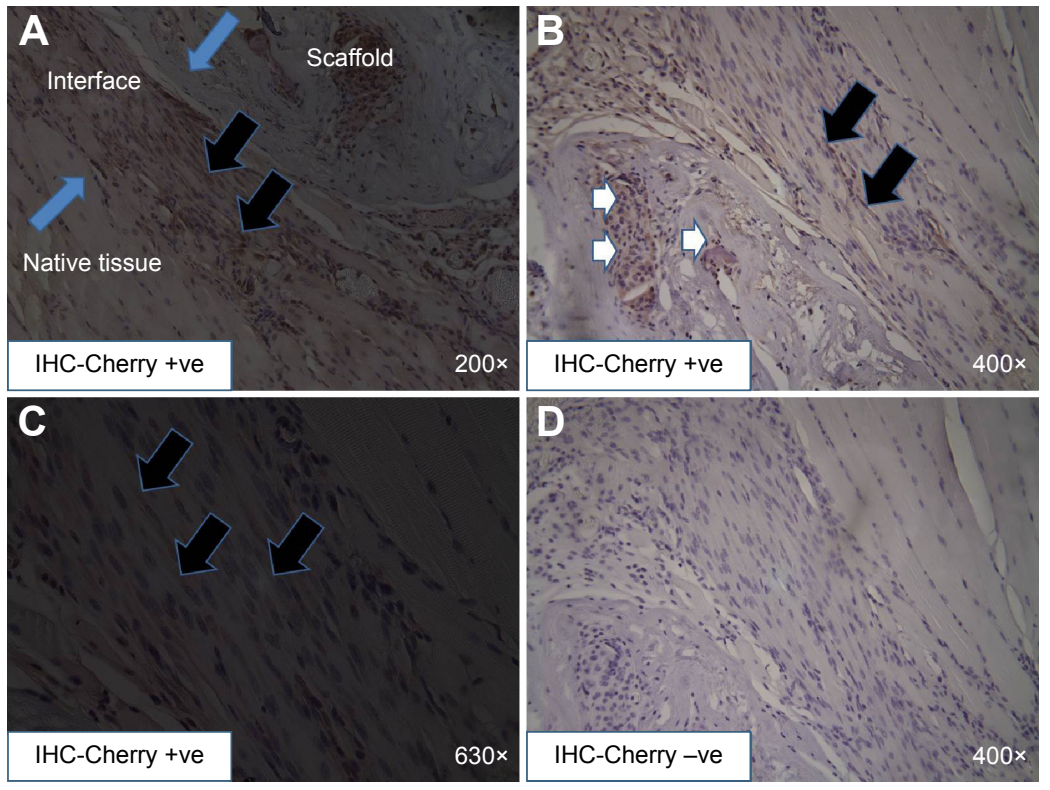

Figure 6 Identification of donor MDCs in retrieved engineered muscles repaired with TEMR-MDC constructs.

Notes: Lentivirus expressing Cherry reporter was transduced into MDCs prior to seeding on to BAM scaffolds. TEMR-lenti-Cherry-MDC ( $\mathrm{n}=8$ )-implanted engineered muscles were retrieved 2 months postrepair and processed for paraffin embedding. Immunohistochemistry (IHC) staining was performed on sections with anti-Cherry antibody. A depicts an overview of IHC cherry positive staining at the interface (demarcated by blue arrows) of native tissue and scaffold. Newly regenerating, differentiating, and elongated myofibers incorporating donor MDCs could be detected at low (A) or high magnification (B and $\mathbf{C})$, indicated by black arrows; scaffold also displayed unfused donor MDCs, as indicated by white arrows (B). Negative controls (D) from adjacent sections were displayed to confirm the specificity of anti-Cherry antibody staining. IHCCherry -ve, negative control, done by omitting primary (Cherry) antibody; IHC-Cherry +ve, positive staining obtained upon using primary (Cherry) antibody. IHC-Cherry -ve and IHC-Cherry +ve indicate color development by IHC is specific, ie, there is no color development in the absence of primary antibody.

Abbreviations: MDC, muscle-derived progenitor cell; TEMR, tissue engineered muscle repair; BAM, bladder acellular matrix; TEMR-lenti-Cherry-MDC, tissue engineered muscle repair construct created with seeding MDCs that were labeled with lentivirally expressing Cherry reporter; IHC, immunohistochemistry.
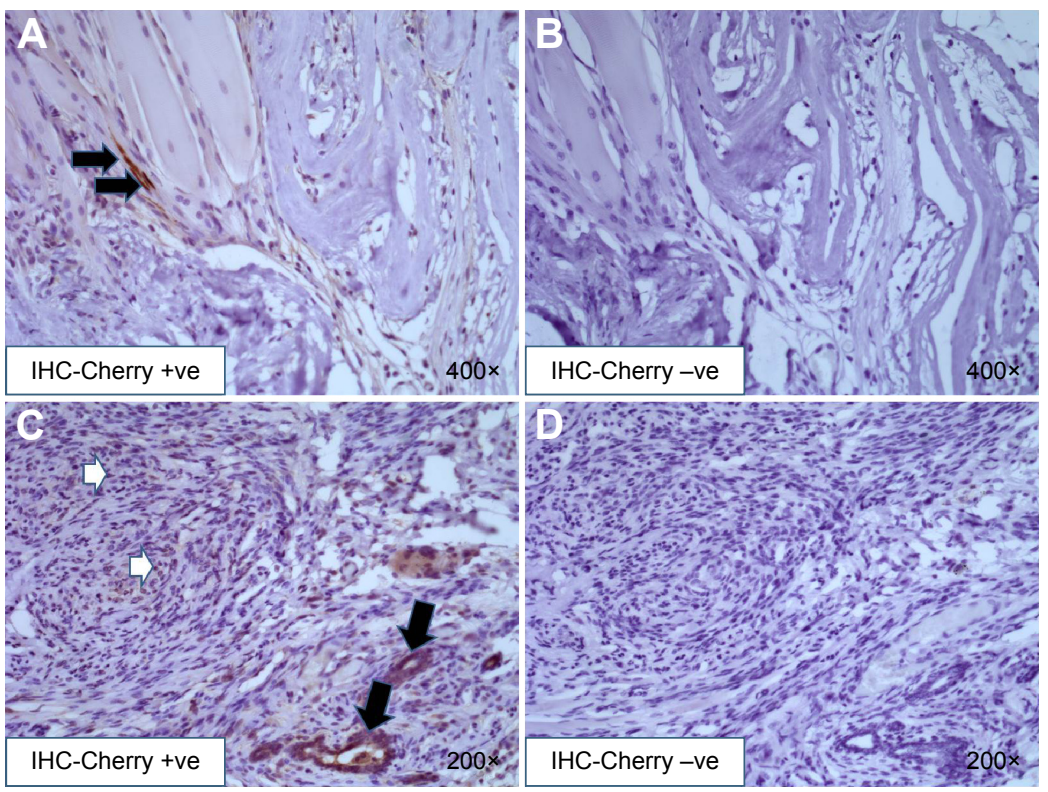

Figure 7 Identification of donor ADSCs in retrieved engineered muscles repaired with TEMR-ADSC constructs.

Notes: Lentivirus expressing Cherry reporter was transduced into ADSCs prior to seeding on to BAM scaffolds. TEMR-lenti-Cherry-ADSC ( $\mathrm{n}=8$ )-implanted engineered muscles were retrieved 2 months postrepair and processed for paraffin embedding. Immunohistochemistry (IHC) staining was performed on sections with anti-Cherry antibody. Occasionally, hybrid myofibers incorporating donor ADSCs could be detected in differentiating, elongated newly forming myofibers at the interface, indicated by black arrows (A). B depicts corresponding negative control from an adjacent section. (C) A large part of the scaffold, however, displays unfused donor ADSCs (white arrows). Interestingly, unfused donor ADSCs were also detected surrounding putative new blood vessel-like structures (black arrows) indicating that ADSCs can potentially participate or enhance vascularization. D depicts corresponding negative control from an adjacent section. IHC-Cherry -ve, negative control, done by omitting primary (Cherry) antibody; IHC-Cherry +ve, positive staining obtained upon using primary (Cherry) antibody. IHC-Cherry -ve and IHC-Cherry +ve indicate color development by $\mathrm{IHC}$ is specific, ie, there is no color development in the absence of primary antibody.

Abbreviations: ADSC, adipose-derived stem cell; TEMR, tissue engineered muscle repair; BAM, bladder acellular matrix; TEMR-lenti-Cherry-ADSC, tissue engineered muscle repair construct created with seeding ADSCs that were labeled with lentivirally expressing Cherry reporter; IHC, immunohistochemistry. 
developmental stages of cell cycle. TEMR-MDC constructs displayed a differentiated phenotype consisting of myotubes interspersed with some unfused myogenic cells as previously reported, ${ }^{16,17}$ whereas TEMR-ADSC constructs primarily displayed an undifferentiated phenotype. ADSCs on BAM scaffolds were left undifferentiated because prior reports suggested that ADSC differentiation best occurred only in the presence of cocultured muscle cells. ${ }^{31}$ Additionally, we intended to take full advantage of trophic effects of undifferentiated ADSCs.

Histological analysis of both TEMR-ADSC and TEMRMDC groups demonstrated evidence of muscle repair, including hallmarks of neotissue formation such as cell fusion and myofiber formation. Notably, nascent striated muscle fiber formation was observed on the engineered side of the scaffold, indicating that repair was mediated by TEMR-MDC and TEMR-ADSC constructs. Regenerating muscle fibers also expressed both structural proteins (as revealed by IHC staining of titin, MHC) in a sarcomeric arrangement and functional proteins necessary for excitation-contraction coupling (as revealed by IHC staining of Junctophilin and ryanodine receptors).

Although the definitive mechanism for TEMR-ADSC construct-based VML repair is not yet clear, the apparent lack of equivalent regeneration in the NR and R-S groups (groups lacking donor cells), as was demonstrated from prior reports, ${ }^{16,17}$ indicates the vital role played by ADSCs in this process. Scaffolds seeded with either MDCs or ADSCs displayed signs of scaffold remodeling and neotissue formation (Figures 2 and 3). Conversely, histological analysis of R-S explants displayed large acellular regions and minimal scaffold remodeling (data not shown). Results from our donor cell survival analysis (Figures 6 and 7) suggest that similar to MDCs, some donor ADSCs also survived in retrieved engineered muscles at 2 months postimplantation. While direct contribution of donor ADSCs to new muscle fiber formation by way of forming new hybrid myofibers incorporating donor cells appears low, compared to donor MDCs, they may compensate for this lower efficiency in tissue regeneration with participation in vascularization and also through paracrine signaling and immunomodulation.

Literature evidence suggests that ADSC transplantation in 24-week-old dystrophin-deficient mice improved muscle strength and resistance to fatigue. ${ }^{37}$ Moreover, an increase in fiber cross-sectional area, increase in the number of fibers with centralized nuclei, and augmentation of myogenin content were also observed. ${ }^{37}$ Additionally, ADSC-treated muscles displayed a reduction in muscle content of tumor necrosis factor- $\alpha$, interleukin (IL)-6, and oxidative stress.
The level of transforming growth factor- $\beta 1$ (an important factor favoring fibrosis) was lowered, whereas the levels of vascular endothelial growth factor, IL-10, and IL-4 were increased by ADSC treatment. ${ }^{37}$ An increase in markers of macrophage M1 (CD11 and F4-80) and a decrease in $\mathrm{T}$ lymphocyte marker (CD3) and arginase-1 were also observed in ADSCs-treated dystrophic muscle. No change in inducible nitric oxide synthase expression was detected. Moreover, increased phosphorylation of Akt, p70S6k, and 4E-BP1 was found in dystrophic muscles treated with ADSC. These results suggest that ADSC transplantation modulates inflammation and improves muscle tissue regeneration, as was demonstrated in dystrophin-deficient mice. ${ }^{37}$ While our study did not inject ADSCs directly into injured muscle, the proximity of ADSC-seeded TEMR constructs with the injury bed and the host cells may provide a conducive environment to neotissue formation and its integration with the host tissue. Although donor cell participation in new myofiber formation was relatively poor (Figure 7), the presence of cellular components in the initial stages of inflammatory phase and scaffold remodeling and participation in vascularization may have established a favorable environment for migration and differentiation of host cells by secretion of extracellular matrix components and soluble factors from donor ADSCs. ${ }^{38}$ Since prior literature suggests that ADSCs in the undifferentiated phenotype may provide paracrine signaling through their trophic effects on the protection, survival, and differentiation of variety of endogenous cells/tissues, ${ }^{38,39}$ some degree of regeneration could be potentially attributed to host muscle cells and components of inflammatory process at the site of surgical defect and the interplay of soluble factors between host cells and ADSCs present on BAM. ${ }^{37}$ In this context, it is noteworthy to mention that prior literature evidence also suggests that soluble factors secreted by myogenic cells are sufficient to promote the expression of muscle-specific proteins in ADSCs cocultured with myogenic cells, ${ }^{33,40}$ indicating that tissue regeneration mediated by ADSCs could also play a role.

It remains to be determined whether a mixture of ADSCs and MDCs together to generate TEMR constructs would offer additional advantages over either cell type alone. It is conceivable that since VML injuries are usually accompanied by loss of not only skeletal muscle but also associated blood vessels and nerve connections, any additional functionality capable of accelerating angiogenesis and innervation while new muscle fibers are being regenerated might confer additional benefits over constructs composed of biomaterials and muscle progenitors alone, thereby improving therapeutic outcomes. ${ }^{37}$ While research work in the group of Badylak 
shows that an acellular scaffold alone may have beneficial effect by recruitment of satellite stem cells and other factors that facilitate skeletal muscle regeneration, ${ }^{10}$ in complex VML injuries, the availability and recruitment of such cells may be more challenging. ${ }^{41}$ Therefore, approaches supplying exogenous cells may offer significant and rapid functional recovery, as demonstrated by recent reports. ${ }^{16-18} \mathrm{We}$ speculate that TEMR-ADSCs may facilitate rapid angiogenesis and new muscle tissue formation via direct and indirect mechanisms. In summary, we have demonstrated that TEMR constructs generated with ADSCs displayed histologically similar regeneration potential compared to TEMR-MDC constructs.

\section{Acknowledgment}

The author thanks Mrs Manasi Vadhavkar, Mr Benjamin Rowe, and Dr Weixien Zhao for their help in histology and mouse VML surgeries.

\section{Disclosure}

The author reports no conflicts of interest in this work.

\section{References}

1. Birnbaum KD, Alvarado AS. Slicing across kingdoms: regeneration in plants and animals. Cell. 2008;132:697-710.

2. Tsonis PA. Limb Regeneration. New York, NY: Cambridge University Press; 1996. ISBN 978-0-521-44149-0.

3. Lanza R, Langer R, Vacanti JP. Principles of Tissue Engineering. San Diego, CA: Academic press; 2011.

4. Carlson BM, Faulkner JA. The regeneration of skeletal muscle fibers following injury: a review. Med Sci Sports Exerc. 1983;15:187-198.

5. Ciciliot S, Schiaffino S. Regeneration of mammalian skeletal muscle: Basic mechanisms and clinical implications. Curr Pharm Des. 2010;16: 906-914.

6. White TP, Devor ST. Skeletal muscle regeneration and plasticity of grafts. Exerc Sport Sci Rev. 1993;21:263-295.

7. Norris BL, Kellam JF. Soft-tissue injuries associated with high-energy extremity trauma: principles of management. J Am Acad Orthop Surg. 1997;5:37-46

8. Grogan BF, Hsu JR; Skeletal trauma research consortium. Volumetric muscle loss. J Am Acad Orthop Surg. 2011;19(suppl 1):S35-S37.

9. Lawson R, Levin LS. Principles of free tissue transfer in orthopedic practice. J Am Acad Orthop Surg. 2007;15:290-299.

10. Sicari BM, Rubin JP, Dearth CL, et al. An acellular biologic scaffold promotes skeletal muscle formation in mice and humans with volumetric muscle loss. Sci Transl Med. 2014;6:234ra58.

11. Mase VJ, Hsu JR, Wolf SE, et al. Clinical application of an acellular biologic scaffold for surgical repair of a large, traumatic quadriceps femoris muscle defect. Orthopedics. 2010;33:511.

12. Montarras D, Morgan J, Collins C, et al. Direct isolation of satellite cells for skeletal muscle regeneration. Science. 2005;309:2064-2067.

13. Chang H, Yoshimoto M, Umeda K, et al. Generation of transplantable, functional satellite-like cells from mouse embryonic stem cells. FASEB J. 2009;23:1907-1919.

14. Mitchell KJ, Pannérec A, Cadot B, et al. Identification and characterization of a non-satellite cell muscle resident progenitor during postnatal development. Nat Cell Biol. 2010;12:257-266.
15. Dellavalle A, Sampaolesi M, Tonlorenzi R, et al. Pericytes of human skeletal muscle are myogenic precursors distinct from satellite cells. Nat Cell Biol. 2007;9:255-267.

16. Machingal MA, Corona BT, Walters TJ, et al. A tissue-engineered muscle repair construct for functional restoration of an irrecoverable muscle injury in a murine model. Tissue Eng Part A. 2011;17:2291-2303.

17. Corona BT, Machingal MA, Criswell T, et al. Further development of a tissue engineered muscle repair (TEMR) construct in vitro for enhanced functional recovery following implantation in vivo in a murine model of volumetric muscle loss (VML) injury. Tissue Eng Part A. 2012; 18:1213-1228.

18. Corona BT, Ward CL, Baker HB, Walters TJ, Christ GJ. Implantation of in vitro tissue engineered muscle repair constructs and bladder acellular matrices partially restore in vivo skeletal muscle function in a rat model of volumetric muscle loss injury. Tissue Eng Part A. 2014;20: 705-715.

19. Tedesco FS, Dellavalle A, Diaz-Manera J, Messina G, Cossu G. Repairing skeletal muscle: regenerative potential of skeletal muscle stem cells. J Clin Invest. 2010;120:11-19.

20. Merritt EK, Cannon MV, Hammers DW, et al. Repair of traumatic skeletal muscle injury with bone-marrow-derived mesenchymal stem cells seeded on extracellular matrix. Tissue Eng Part A. 2010;16: 2871-2881.

21. De Bari C, Dell'Accio F, Vandenabeele F, Vermeesch JR, Raymackers JM, Luyten FP. Skeletal muscle repair by adult human mesenchymal stem cells from synovial membrane. J Cell Biol. 2003;160:909-918.

22. Pittenger MF, Mackay AM, Beck SC, et al. Multilineage potential of adult human mesenchymal stem cells. Science. 1999;284:143-147.

23. Uezumi A, Fukada S, Yamamoto N, Takeda S, Tsuchida K. Mesenchymal progenitors distinct from satellite cells contribute to ectopic fat cell formation in skeletal muscle. Nat Cell Biol. 2010;12:143-152.

24. Yin H, Price F, Rudnicki MA. Satellite cells and the muscle stem cell niche. Physiol Rev. 2013;93:23-67.

25. Boonen KJM, Rosaria-Chak KY, Baaijens FPT, van der Schaft DWJ, Post MJ. Essential environmental cues from the satellite cell niche: optimizing proliferation and differentiation. Am J Physiol Cell Physiol. 2009;296:C1338-C1345.

26. Darabi R, Santos FN, Filareto A, et al. Assessment of the myogenic stem cell compartment following transplantation of Pax3/Pax7induced embryonic stem cell-derived progenitors. Stem Cells. 2011;29: 777-790.

27. Page RL, Malcuit C, Vilner L, et al. Restoration of skeletal muscle defects with adult human cells delivered on fibrin microthreads. Tissue Eng Part A. 2011;17:2629-2640.

28. Kocaefe Ç, Balci D, Balci Hayta B, Can A. Reprogramming of human umbilical cord stromal mesenchymal stem cells for myogenic differentiation and muscle repair. Stem Cell Rev Rep. 2010;6:512-522.

29. Berry SE, Liu J, Chaney EJ, Kaufman SJ. Multipotential mesoangioblast stem cell therapy in the $\mathrm{mdx} / \mathrm{utrn}^{-/}$mouse model for duchenne muscular dystrophy. Regen Med. 2007;2:275-288.

30. Zuk P. Adipose-derived stem cells in tissue regeneration: a review. ISRN Stem Cells. 2013;2013:1-35.

31. Zuk PA, Zhu M, Ashjian P, et al. Human adipose tissue is a source of multipotent stem cells. Mol Biol Cell. 2002;13:4279-4295.

32. Kingham PJ, Kalbermatten DF, Mahay D, Armstrong SJ, Wiberg M, Terenghi G. Adipose-derived stem cells differentiate into a schwann cell phenotype and promote neurite outgrowth in vitro. Exp Neurol. 2007; 207:267-274.

33. Di Rocco G, Iachininoto MG, Tritarelli A, et al. Myogenic potential of adipose-tissue-derived cells. J Cell Sci. 2006;119:2945-2952.

34. Moon DG, Christ G, Stitzel JD, Atala A, Yoo JJ. Cyclic mechanical preconditioning improves engineered muscle contraction. Tissue Eng Part A. 2008;14:473-482.

35. Schiaffino S, Partridge T. Skeletal Muscle Repair and Regeneration. Dordrecht: Springer Science \& Business Media; 2008. ISBN 978-14020-6768-6 (e-book). 
36. Corona BT, Wu X, Ward CL, McDaniel JS, Rathbone CR, Walters TJ. The promotion of a functional fibrosis in skeletal muscle with volumetric muscle loss injury following the transplantation of muscle-ECM. Biomaterials. 2013;34:3324-3335.

37. Da Justa Pinheiro CH, de Queiroz JC, Guimarães-Ferreira L, et al. Local injections of adipose-derived mesenchymal stem cells modulate inflammation and increase angiogenesis ameliorating the dystrophic phenotype in dystrophin-deficient skeletal muscle. Stem Cell Rev. 2012;8(2):363-374.

38. Salgado AJ, Reis RL, Sousa NJ, Gimble JM. Adipose tissue derived stem cells secretome: soluble factors and their roles in regenerative medicine. Curr Stem Cell Res Ther. 2010;5:103-110.
39. Aurora AB, Olson EN. Immune modulation of stem cells and regeneration. Cell Stem Cell. 2014;15:14-25.

40. Mizuno $H$. The potential for treatment of skeletal muscle disorders with adipose-derived stem cells. Curr Stem Cell Res Ther. 2010;5: $133-136$.

41. Garg K, Ward CL, Rathbone CR, Corona BT. Asynchronous inflammation and myogenic cell migration limit muscle tissue regeneration mediated by acellular scaffolds. Inflamm Cell Signal. 2015;2:e530.
International Journal of Nanomedicine

\section{Publish your work in this journal}

The International Journal of Nanomedicine is an international, peerreviewed journal focusing on the application of nanotechnology in diagnostics, therapeutics, and drug delivery systems throughout the biomedical field. This journal is indexed on PubMed Central, MedLine, CAS, SciSearch $®$, Current Contents $® /$ Clinical Medicine,

\section{Dovepress}

Journal Citation Reports/Science Edition, EMBase, Scopus and the Elsevier Bibliographic databases. The manuscript management system is completely online and includes a very quick and fair peer-review system, which is all easy to use. Visit http://www.dovepress.com/ testimonials.php to read real quotes from published authors.

Submit your manuscript here: http://www.dovepress.com/international-journal-of-nanomedicine-journal 